\title{
Desafios na Profilaxia do Tromboembolismo Venoso: Abordagem do Paciente Crítico*
}

\author{
Challenges in Prevention Venous \\ Thromboembolism: Critical ill Patient approach
}

Marcelo Andrade Ribeiro ${ }^{1}$, Pedro Garbes Netto², Silvia Gelas Lage ${ }^{3}$

\section{RESUMO}

JUSTIFICATIVA E OBJETIVOS: O tromboembolismo venoso (TEV), que inclui a trombose venosa profunda (TVP) e o tromboembolismo pulmonar (TEP) são complicações comuns em pacientes críticos. A ocorrência de TEV acarreta um substancial aumento da morbimortalidade dos pacientes internados em unidades de terapia intensiva (UTI).

CONTEÚDO: A maioria dos pacientes críticos apresenta alto risco para ocorrência de complicações tromboembólicas, entretanto, a prevenção do TEV muitas vezes não é realizada de maneira adequada para este grupo de pacientes. A heparina de baixo peso molecular (HBPM) parece ser o método mais eficiente para a prevenção do TEV em pacientes de UTI. Entretanto, é patente a escassez de estudos voltados para esta população, cujas particularidades levam a recomendações específicas em relação ao diagnóstico e tratamento.

1. Médico Assistente da Unidade de Terapia Intensiva do Instituto do Coração (InCor) do HCFMUSP

2. Médico do Serviço de Infectologia da Universidade Federal Fluminense; Diretor Médico do Instituto BioChimico, Rio de Janeiro, RJ 3. Professora Associada da Faculdade de Medicina da Universidade de São Paulo; Diretora da Unidade Clínica de Terapia Intensiva - Instituto do Coração (InCor) do HCFMUSP.

*Recebido da Unidade de Terapia Intensiva do Instituto do Coração (InCor) do Hospital das Clínicas da Faculdade de Medicina da Universidade de São Paulo (USP), São Paulo, SP.

Apresentado em 17 de maio de 2006

Aceito para publicação em 11 de setembro de 2006

Endereço para correspondência:

Dra. Silvia Gelas Lage

Av. Dr. Enéas de Carvalho Aguiar, 44 - Sala 10, $2^{\circ} \mathrm{A} / \mathrm{Bloco}$ II

05403-900 São Paulo, SP

Fone (011) 3069.5697 / 5397 - Fax (011) 3069.5397

E-mail: sglage@incor.usp.br, marcandrib@yahoo.com.br

(C)Associação de Medicina Intensiva Brasileira, 2006
CONCLUSÕES: Esta revisão faz uma análise do risco, discute os principais trabalhos publicados a respeito da profilaxia e sugere estratégias para a diminuição da ocorrência de TEV nos pacientes críticos.

Unitermos: paciente crítico, prevenção de TVP, trombose venosa profunda, tromboembolismo pulmonar

\section{SUMMARY}

BACKGROUND AND OBJECTIVES: Venous thromboembolism (VTE), with includes deep vein thrombosis (DVT) and pulmonary embolism (PE), is a common complication in critically ill patients, resulting in high morbidity and mortality.

CONTENTS: Most patients treated in intensive care units (ICU) face a high risk of thromboembolic complications. Despite these considerations, the prevention of VTE may not be as high a priority in ICU patients as it is in other high-risk patient groups. Low molecular weight heparin (LMWH) may be the optimal prophylaxis in most ICU patients, but there is a lack of sufficient data including the paucity of VTE consensus and guidelines documents pertaining to critically ill patients.

CONCLUSIONS: This article reviews background, current options, and recommendations regarding VTE in intensive care population emphasizing special diagnostic and treatment considerations in the ICU setting. Key Words: deep vein thrombosis, ICU patients, pulmonary embolism, VTE prevention

\section{INTRODUÇÃO}

O tromboembolismo venoso (TEV) representa um espectro de doenças que contempla a trombose venosa profunda (TVP) a trombose associada a cateteres venosos centrais (trombose-CVC) e o tromboembolismo pulmonar (TEP). Apesar do conhecimento da fisiopatologia do TEV remontar ao inicio do século XIX 
- quando o patologista alemão Rudolf Virchow descreveu a tríade clássica composta por estase venosa, lesão endotelial e hipercoagulabilidade - esta condição ainda é uma das principais causas de morte em todo o mundo ${ }^{1-4}$.

O TEV é relativamente comum em pacientes hospitalizados e tem grande impacto sobre a morbimortalidade, tanto em pacientes cirúrgicos quanto clínicos ${ }^{5}$. Até meados dos anos 1990 a maioria dos estudos avaliou a incidência de TEV apenas em populações cirúrgicas fazendo com que muito mais ênfase fosse dada à necessidade de profilaxia para este grupo de pacientes.

Recentemente, estudos clínicos aleatórios envolvendo pacientes internados devido a doenças puramente clínicas, demonstraram que a ocorrência de TEV é similar à encontrada em pacientes cirúrgicos ${ }^{6,7}$. Em concordância, já foi demonstrado que mais da metade dos casos de TEV sintomáticos acometem pacientes sem nenhuma afecção cirúrgica ${ }^{8}$. Apesar disso, dados do registro RIETE ${ }^{9}$ mostraram que a profilaxia do TEV muitas vezes ainda é esquecida para os pacientes clínicos. Este registro incluiu mais de 6000 pacientes, todos com TEV comprovado, sendo que apenas $28 \%$ dos pacientes clínicos receberam profilaxia, comparados com $67 \%$ dos pacientes cirúrgicos.

Visando corrigir esta distorção as mais recentes diretrizes enfatizaram a necessidade de atenção para a profilaxia nos pacientes clínicos. Entretanto, é patente a escassez de recomendações específicas voltadas para um grupo com risco particularmente elevado: o paciente crítico.

O paciente crítico deve ser alvo de atenção diferenciada não somente pelo alto risco, mas também em função da multiplicidade de variáveis que podem influenciar na decisão a respeito da profilaxia. Muitas vezes, a definição de uma conduta pode se tornar extremamente difícil, envolvendo considerações específicas em relação ao risco de eventos hemorrágicos. Neste grupo de pacientes a avaliação da relação risco-benefício apresenta particularidades e as recomendações atuais para a profilaxia do TEV não podem ser simplesmente extrapoladas para o ambiente de terapia intensiva.

A presente revisão teve como base um levantamento realizado no banco de dados da PubMed na qual os termos em língua inglesa deep vein trombosis, ICU patients, pulmonary embolism e VTE prevention foram utilizados para se determinar as publicações pertinentes referentes ao tema.

\section{AVALIAÇÃO DO RISCO PARA OCORRÊNCIA DE TEV}

A TVP e o TEP contribuem significativamente para aumentar a morbimortalidade de pacientes críticos $^{10,11}$. Em pacientes internados em UTI a ocorrência de TVP é alta ${ }^{12}$. Os pacientes que não recebem profilaxia constituem um grupo de alto risco, com incidência variando de $25 \%$ a $31 \%$. Entretanto, quando a profilaxia é adequada, a taxa de TVP diminui pela metade ${ }^{5,13-15}$.

O tromboembolismo pulmonar (TEP) deve ser encarado como complicação de uma trombose venosa, formada, na maioria dos casos, no sistema venoso profundo dos membros inferiores. No ocidente, sua incidência na população geral é estimada em 5/10.000 pacientes; com mortalidade quatro vezes maior, quando o tratamento não é instituído ${ }^{15}$. Entretanto, o diagnóstico é freqüentemente subestimado, sendo que apenas $40 \%$ dos casos de embolia pulmonar diagnosticado em necropsia foram suspeitados clinicamente ${ }^{7}$. O TEP tem sido reportado como causa de morte em $1 \%$ a $12 \%$ dos pacientes críticos e a utilização de profilaxia nestes casos ainda é bastante irregular, apesar da maioria ter dois ou mais fatores de risco conhecidos ${ }^{16}$.

Os principais fatores de risco para o desenvolvimento de TEV nos pacientes críticos estão apresentados no quadro 1.

Quadro 1 - Fatores de Risco para a Ocorrência de TEV em Pacientes Críticos

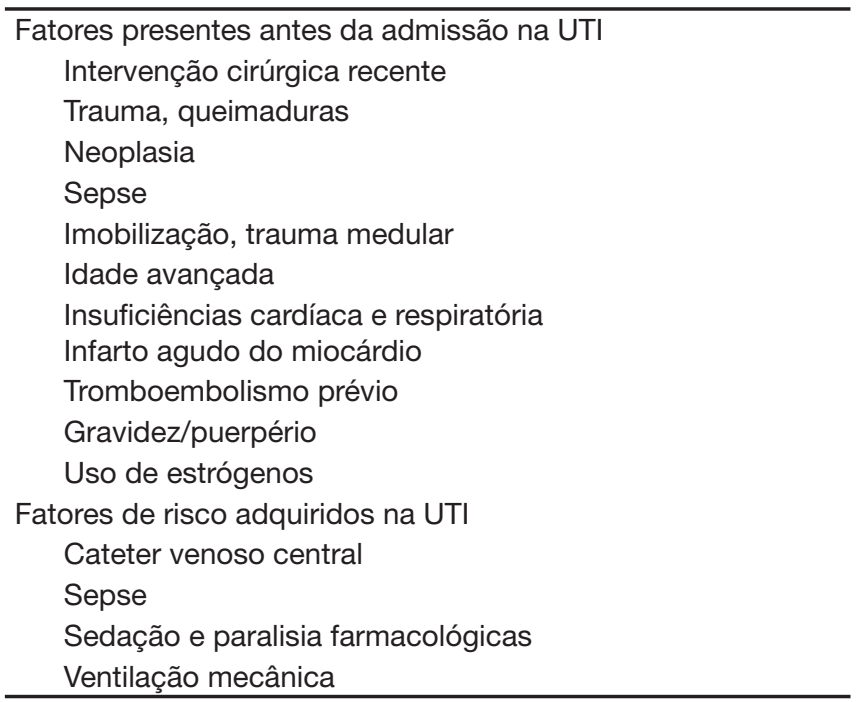

Devido à coexistência de múltiplos fatores de risco em associação com a baixa acurácia do exame físico, não é raro que TVP não suspeitada possa estar presente mesmo antes da internação na UTI. Quatro séries, envolvendo um total de 729 pacientes críticos, demonstraram 
que, quando se utilizou a ultra-sonografia com Doppler, realizada nos membros inferiores logo na admissão, o diagnóstico de TVP foi realizado em $6,4 \%$ dos casos, todos até então insuspeitos ${ }^{13,17-19}$.

\section{PROFILAXIA DO TEV}

Um grande número de estudos aleatórios, envolvendo pacientes críticos, demonstrou que a utilização de heparina é o método mais eficiente para prevenção do $\mathrm{TEV}^{1,6,7,10,13}$. Para os pacientes de alto risco para ocorrência de TEV, conforme especificado na tabela 1, a heparina de baixo peso molecular (HBPM) parece ser mais eficiente do que a heparina não fracionada $(\mathrm{HNF})^{2}$. Além disso, a HBPM apresenta maior comodidade posológica - apenas uma aplicação diária - e a ocorrência de plaquetopenia induzida por heparina é substancialmente menor quando comparada a HNF'1. Todos os pacientes críticos são considerados, em relação a ocorrência de TEV, no mínimo de risco moderado. Para estes pacientes a decisão em relação ao tipo de heparina a ser utilizada deve levar em consideração a avaliação do risco de sangramento conforme mostra a tabela 1.

Tabela 1 - Recomendações para Profilaxia de TEV em Pacientes Críticos

\begin{tabular}{|c|c|c|}
\hline $\begin{array}{l}\text { Riscos de } \\
\text { Sangramento }\end{array}$ & $\begin{array}{l}\text { Riscos de } \\
\text { Eventos Trom- } \\
\text { boembólicos }\end{array}$ & $\begin{array}{l}\text { Tipo de Profilaxia Recomenda- } \\
\text { da }\end{array}$ \\
\hline Baixo & Moderado & $\begin{array}{l}\text { Heparina não fracionada } \\
5000 \text { U, por via subcutânea a } \\
\text { cada } 12 / 12 \text { horas }\end{array}$ \\
\hline Baixo & Alto risco* & $\begin{array}{l}\text { HBPM (dose varia com o tipo } \\
\text { de HBPM utilizada) }\end{array}$ \\
\hline Alto & Moderado & Profilaxia mecânica com CPI \\
\hline Alto & Alto risco & Profilaxia mecânica com CPI \\
\hline
\end{tabular}

$\mathrm{CPI}=$ compressão pneumática intermitente; HBPM = heparina de baixo peso molecular.

"Alto Risco" definido pela ocorrência de pelo menos um destes fatores: trauma, lesão medular, pós-operatório de cirurgia ortopédica, neoplasias.

O risco de sangramento deve ser avaliado de maneira individualizada e a definição (alto ou baixo) fica a critério do intensivista.

\section{METODOS NÃO FARMACOLÓGICOS}

Em relação à profilaxia com métodos mecânicos, foi demonstrada diminuição na ocorrência de TEV em pacientes não críticos, com a utilização da compressão pneumática intermitente $(\mathrm{CPI})^{2}$. Entretanto, a maioria dos estudos aleatórios comparando $\mathrm{CPI}$ versus profilaxia com heparina mostraram superioridade do método farmacológico ${ }^{16}$.

Quando se avalia de maneira isolada apenas a população de pacientes críticos a literatura é extremamente escassa em relação aos métodos mecânicos de profila- xia. Recentemente foi publicado um estudo que avaliou a eficácia do uso de CPI em pacientes com alto risco para a ocorrência de TEV internados em UTI cirúrgica ${ }^{20}$. Este estudo incluiu 38 pacientes com contra-indicação absoluta para o uso de heparina. Todos os pacientes foram submetidos, na ocasião da alta da UTI, à realização de ultra-sonografia com Doppler nos membros inferiores, sendo que nenhum apresentou evidência de TVP. Apesar de já ter sido demonstrado que em pacientes críticos vitimas de trauma crânio-encefálico ou lesão raqui-medular o uso de CPI tem a mesma eficácia que a profilaxia com HBPM para a prevenção da ocorrência de $\mathrm{TEV}^{21}$ estes métodos ainda não foram adequadamente investigados em pacientes críticos não cirúrgicos.

\section{MONITORIZAÇÃO COM A DOSAGEM DO FATOR ANTI-Xa}

A utilização da dosagem do fator anti-Xa para monitoramento da eficácia da HBPM nos pacientes críticos foi pouco estudada. Grande atenção tem sido dada aos pacientes com insuficiência renal (IR), tendo em vista o potencial risco de acúmulo do fármaco ${ }^{22-25}$. Apesar da pouca evidência científica envolvendo o tema, a monitorização com a dosagem do fator anti-Xa tem sido recomendada para dois grupos: pacientes com insuficiência renal avançada (definida por depuração de creatinina menor que $30 \mathrm{~mL} / \mathrm{min}$ ) e pacientes obesos ${ }^{26}$.

Entretanto, outras situações comumente encontradas em pacientes críticos - como o baixo débito cardíaco e o edema do tecido subcutâneo - potencialmente podem influenciar na biodisponibilidade da HBPM. A hipótese de que a diminuição da perfusão tecidual poderia interferir na absorção da HBPM, tendo em vista que o fármaco é administrado por via subcutânea, ainda não foi adequadamente investigada. Em relação ao potencial prejuízo da absorção do fármaco devido ao edema do tecido subcutâneo, um estudo recente realizado com pacientes críticos, mostrou que a presença de edema subcutâneo não interfere na atividade da HBPM medida através da dosagem do fator anti-Xa ${ }^{27}$. Além deste, apenas outros dois estudos aleatórios utilizaram a dosagem sérica do fator anti-Xa para avaliação da eficácia da profilaxia em pacientes $\operatorname{críticos}^{28,29}$. Em ambos, o enfoque foi o risco do acúmulo do fármaco nos casos de insuficiência renal, sendo que nenhum deles contemplou pacientes com baixo débito cardíaco. Recentemente foi publicado um estudo mostrando que a monitorização do uso da HBPM com a dosagem do fator anti-Xa em pacientes críticos parece ser útil, sobretudo naqueles com baixo debito cardíaco ${ }^{30}$. 


\section{RESUMO DAS RECOMENDAÇÕES PARA PROFILA- XIA DO TEV EM PACIENTES CRÍTICOS}

As principais recomendações sobre a profilaxia do TEV para os pacientes críticos são:

1) Com raras exceções métodos de prevenção devem ser instituídos para todos os pacientes internados em UTI; 2) Já na admissão os pacientes devem ter o risco de eventos tromboembólicos avaliado e a profilaxia deve ser iniciada o mais breve possível;

3) As decisões a respeito do tipo de profilaxia (mecânica ou farmacológica) devem ser individualizadas, levandose em consideração o risco de sangramento e de eventos tromboembólicos (Tabela 1). Em geral, é preferível o uso de prevenção farmacológica, entretanto, para os pacientes com alto risco de sangramento a profilaxia com métodos mecânicos (preferencialmente CPI) deve ser inicialmente utilizada. $O$ risco de sangramento deve ser avaliado diariamente e, uma vez diminuído, a prevenção farmacológica deve ser instituída.

4) O uso rotineiro de ultra-sonografia com Doppler ou qualquer outro método de avaliação objetiva para pacientes assintomáticos adequadamente tratados não é recomendado. Entretanto, para pacientes de alto risco que não receberam profilaxia adequada antes ou durante a internação em UTI, uma avaliação com USG Doppler deverá ser realizada. Da mesma forma pacientes com sinais clínicos sugestivos de TVP devem ser submetidos à realização da USG com Doppler nos membros inferiores;

5) O controle do uso da HBPM, através da dosagem do fator anti-Xa, deve ser realizado em pacientes obesos e naqueles com IR avançada (depuração de creatinina menor que $30 \mathrm{~mL} / \mathrm{min}$ ). Em relação a paciente com diminuição da perfusão tecidual (como em situações de baixo débito cardíaco), a necessidade do controle laboratorial ainda não foi adequadamente investigada, e o uso rotineiro da avaliação com o fator anti-Xa não é recomendado para este grupo de pacientes.

\section{REFERÊNCIAS}

01. International Consensus Statement: prevention of venous thromboembolism. Int Angiol, 1997;16:3-37.

02. Geerts W, Selby R - Prevention of venous thromboembolism in the ICU. Chest, 2003;124:(Suppl1):S357-S363.

03. Hirsch DR, Ingenito EP, Goldhaber SZ - Prevalence of deep venous thrombosis among patients in medical intensive care. JAMA, 1995;274:335-337.

04. Second Thromboembolic Risk Factors (THRiFT II) Consensus Group. Risk of and prophylaxis for venous thromboembolism in hospital patients. Phlebology, 1998;13:87-97.

05. Cade JF - High risk of the critically ill for venous thromboembolism. Crit Care Med, 1982;10:448-450.
06. Bergmann JF, Neuhart E - A multicenter randomized double-blind study of enoxaparin compared with unfractionated heparin in the prevention of venous thromboembolic disease in elderly in-patients bedridden for an acute medical illness. Thromb Haemost, 1996;76:529-534.

07. Leizorovicz A, Cohen AT, Turpie AG et al - Randomized, placebo-controlled trial of dalteparin for the prevention of venous thromboembolism in acutely ill medical patients. Circulation, 2004;110:874-879.

08. Goldhaber SZ, Dunn K, MacDougall RC - New onset of venous thromboembolism among hospitalized patients at Brigham and Women's Hospital is caused more often by prophylaxis failure than by withholding treatment. Chest, 2000;118:1680-1684.

09. Monreal M, Kakkar AK, Caprini JA et al - The outcome alter treatment of venous thromboembolism is different in surgical and acutely ill medical patients. Findings from the RIETE registry. J Thromb Haemost, 2004;2:1892-1898.

10. Hoyt DB, Swegle JR - Deep venous thrombosis in the surgical intensive care unit. Surg Clin North Am, 1991;71:811-830.

11. Davidson BL - Risk assessment and prophylaxis of venous thromboembolism in acutely and/or critically ill patients. Haemostasis, 2000;30:(Suppl2):77-81.

12. Miehsler W, Reinisch W, Valic E et al - Is inflammatory bowel disease an independent and disease specific risk factor for thromboembolism? Gut, 2004;53:542-548.

13. Fraisse F, Holzapfel L, Couland JM et al - Nadroparin in the prevention of deep vein thrombosis in acute decompensated COPD. The Association of Non-University Affiliated Intensive Care Specialist Physicians of France. Am J Respir Crit Care Med, 2000;161:1109-1114.

14. Moser KM, LeMoine JR, Nachtwey FJ et al - Deep venous thrombosis and pulmonary embolism. Frequency in a respiratory intensive care unit. JAMA, 1981;246:1422-1424.

15. Ibrahim EH, Iregui $M$, Prentice $D$ et al - Deep vein thrombosis during prolonged mechanical ventilation despite prophylaxis. Crit Care Med, 2002;30:771-774.

16. Ibarra-Perez C, Lau-Cortes E, Colmenero-Zubiate S et al - Prevalence and prevention of deep venous thrombosis of the lower extremities in high-risk pulmonary patients. Angiology, 1988;39:505-513.

17. Harris LM, Curl GR, Booth FV - Screening for asymptomatic deep vein thrombosis in surgical intensive care patients. J Vasc Surg, 1997;26:764-769.

18. Schonhofer B, Kohler D - Prevalence of deep-vein thrombosis of the leg in patients with acute exacerbation of chronic obstructive pulmonary disease. Respiration, 1998;65:173-177.

19. Geerts WH, Heit JA, Clagett GP et al - Prevention of venous thromboembolism. Chest, 2001;119:(Suppl1):132S-175S.

20. Kurtoglu M, Guloglu R, Ertekin C et al - Intermittent pneumatic compression in the prevention of venous thromboembolism in high-risk trauma and surgical ICU patients. Ulus Travma Acil Cerrahi Derg. 2005;11:38-42.

21. Kurtoglu $\mathrm{M}$, Yanar $\mathrm{H}$, Bilsel $\mathrm{Y}$ et al - Venous thromboembolism prophylaxis after head and spinal trauma: intermittent pneumatic compression devices versus low molecular weight heparin. World J Surg, 2004;28:807-811.

22. Rabbat CG, Cook DJ, Crowther MA - Dalteparin thromboprophylaxis for critically ill medical-surgical patients with renal insufficiency. J Crit Care, 2005;20:357-363.

23. Nagge $\mathrm{J}$, Crowther $\mathrm{M}$, Hirsh $\mathrm{J}$ - Is impaired renal function a contraindication to the use of low-molecular-weight heparin? Arch Intern Med, 2002;162:2605-2609.

24. Goudable C, Saivin S, Houin G et al - Pharmacokinetics of a low molecular weight heparin (Fraxiparine) in various stages of chronic renal failure. $\mathrm{Ne}-$ phron, 1991;59:543-545.

25. Hory B, Claudet MH, Bayrou B et al - Pharmacokinetics of a very low molecular weight heparin in chronic renal failure. Thromb Res, 1991;63:311-317.

26. Johnson PN, Smith KM - Low-molecular-weight heparin use in special populations. Orthopedics, 2004;27:1245-1258.

27. Kapoor M, Kupfer YY, Tessler S - Subcutaneous heparin prophylaxis significantly reduces the incidence of venous thromboembolic events in the critically ill. Crit Care Med 1999;27:(Suppl):A69.

28. Cook DJ, Rocker G, Meade M et al - Prophylaxis of Thromboembolism in Critical Care (PROTECT) Trial: a pilot study. J Crit Care, 2005;20:364-372.

29. Lage S, Carvalho RT, Ribeiro MA et al - Use of anti-Xa factor titration as efficacy marker of enoxaparin in critically ill patients. Crit Care 2006;10:(Suppl1):P161.

30. Rommers MK, van der Lely N, Egberts TC et al - Anti-Xa activity after subcutaneous administration of dalteparin in ICU patients with and without subcutaneous oedema: a pilot study. Crit Care, 2006;10:R93. 